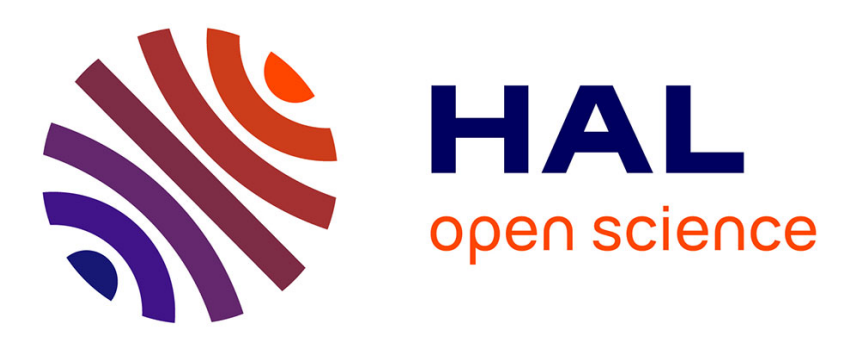

\title{
Patterns of Physical Design Remixing in Online Maker Communities
}

Lora Oehlberg, Wesley Willett, Wendy E. Mackay

\section{To cite this version:}

Lora Oehlberg, Wesley Willett, Wendy E. Mackay. Patterns of Physical Design Remixing in Online Maker Communities. CHI 2015, ACM, Apr 2015, Seoul, South Korea. pp.639-648, 10.1145/2702123.2702175 . hal-01263171

\section{HAL Id: hal-01263171 \\ https://inria.hal.science/hal-01263171}

Submitted on 27 Jan 2016

HAL is a multi-disciplinary open access archive for the deposit and dissemination of scientific research documents, whether they are published or not. The documents may come from teaching and research institutions in France or abroad, or from public or private research centers.
L'archive ouverte pluridisciplinaire HAL, est destinée au dépôt et à la diffusion de documents scientifiques de niveau recherche, publiés ou non, émanant des établissements d'enseignement et de recherche français ou étrangers, des laboratoires publics ou privés.

$$
\text { Copyright }
$$




\section{Patterns of Physical Design Remixing in Online Maker Communities}

\author{
Lora Oehlberg \\ Inria / University of Calgary \\ lora.oehlberg@ucalgary.ca
}

\author{
Wesley Willett \\ Inria / University of Calgary \\ wesley.willett@ucalgary.ca
}

\author{
Wendy E. Mackay \\ Inria \\ wendy.mackay@inria.fr
}

\begin{abstract}
Makers participate in remixing culture by drawing inspiration from, combining, and adapting designs for physical objects. To examine how makers remix each others' designs on a community scale, we analyzed metadata from over 175,000 digital designs from Thingiverse, the largest online design community for digital fabrication. Remixed designs on Thingiverse are predominantly generated designs from Customizer- a builtin web app for adjusting parametric designs. However, we find that these designs do not elicit subsequent user activity and the authors who generate them tend not to contribute additional content to Thingiverse. Outside of Customizer, influential sources of remixing include complex assemblies and design primitives, as well as non-physical resources posing as physical designs. Building on our findings, we discuss ways in which online maker communities could become more than just design repositories and better support collaborative remixing.
\end{abstract}

\section{Author Keywords}

Hacking; Maker Communities; Customization; Remixing;

User Innovation; Collaboration

\section{ACM Classification Keywords}

H.5.m: Information interfaces and presentation (e.g., HCI): Miscellaneous

\section{INTRODUCTION}

Lawrence Lessig [11] argues that we have moved from a "read-only" culture to a "read/write" culture: instead of passively consuming information or products, users now actively participate in creating their own, personalized objects. The Internet and social media has led to an explosion of "remix culture"[6], but until recently, remixing was mostly limited to media content like songs, images, and videos. However, the advent of inexpensive fabrication machines including 3D printers and laser cutters has enabled more consumers to design, create, and remix physical objects that previously required mass manufacture.

Author version of ACM CHI'15 paper.

http://dx.doi.org/10.1145/2702123.2702175
The maker movement [5], an extension of Do-It-Yourself (DIY) culture, has largely grown out of environments like Hackerspaces and FabLabs where hobbyists can create and share their designs. Creating physical objects can require many different kinds of expert knowledge, including how to prepare digital designs for fabrication or operate a fabrication machine. Makers are thus encouraged to borrow and adapt (or remix) each others' designs. Online communities, such as Thingiverse ${ }^{1}$, Youmagine ${ }^{2}$, and Ravelry $^{3}$, form an important piece of this ecosystem, allowing geographically distributed makers to share designs with each other. Visitors to these sites can download publicly available design files and reproduce them using their local fabrication equipment. Some of these communities also record the provenance of remixes, allowing makers to link their work to the earlier designs that inspired them. In Makers, Chris Anderson [1] celebrates the promise of remixing:

The ability to easily "remix" digital files is the engine that drives community. What it offers is an invitation to participate. You don't need to invent something from scratch or have an original idea. Instead, you can participate in a collaborative improvement of existing ideas or designs (p. 74).

In this idealized view of collaboration, makers collectively contribute to the development of new designs by iteratively remixing and refining one another's work. Moreover, this view assumes that remixing serves as an entry point for new makers, who can dissect and build on top of the work of others to bootstrap their own making practice.

While previous examinations of maker communities have looked at remixing anecdotally [20], the rapid growth of online maker communities provides an opportunity to analyze this behavior at a much broader scale. By examining how tens of thousands of makers are actively engaging in remixing on community sites, we can build a more grounded understanding of the impact of remixing on maker communities.

In this paper we investigate how makers remix digital designs for physical objects on Thingiverse, a wellestablished online $3 \mathrm{D}$ printing community. We chose to

\footnotetext{
${ }^{1}$ http://www.thingiverse.com

${ }^{2}$ http://www.youmagine.com

${ }^{3}$ http://www.ravelry.com
} 
focus specifically on Thingiverse-which calls itself "the world's largest 3D printing community"-because detailed metadata about remixing and authorship is available for most of the objects shared on the site since its inception in 2008.

We begin with a review of related work and then present findings from a quantitative analysis of five years of metadata. We examine the impact of Customizer-a parametric design tool within Thingiverse-and also examine non-Customizer designs to explore how users interpret remixing. We identify genres of designs whose remixing behavior manifests itself in unexpected ways. We conclude with specific suggestions for online design repositories and design software that provide better support for remixing and build stronger online maker communities.

\section{RELATED WORK}

This paper examines how a specific online maker community, Thingiverse, supports remixing of physical designs. Our investigation relies on prior studies of maker culture, which provide context for understanding maker behavior and motivations. We also build on prior examinations of remixing practice in other disciplines.

\section{Maker Culture}

Research into maker culture has focused primarily on qualitative studies of individuals' experiences as they participate in maker communities, including their motivations for participation [10], and how being a maker influenced their interactions with materiality [7] and changed their involvement in consumerism [15]. Makers' main motivations for contributing to communities include finding inspiration for future projects, learning new concepts, and receiving feedback on projects [10]. Researchers have profiled specific maker communities and do-it-yourself (DIY) practices, such as IKEA Hacking [18] and Steampunk [19]. These researchers have advocated for the development of new creative tools that "celebrate skilled, creative reuse and customization" [18] or attract "users who are not content to consume but wish to customize, remix, and design for themselves" [19]. Online maker communities already offer basic support for sharing and downloading files for remixing, recording the provenance of design inspirations, and even platforms for easy customization. Most of this previous research has been qualitative, capturing individuals' experiences and motivations for participation, but has yet to provide a community-scale understanding of how makers share and build on one another's ideas.

\section{Remixing}

Not just makers remix; everyday users consistently reuse and re-appropriate mass-produced designs [21]. By remixing others' work, individual makers can learn from others' ideas and more quickly create improved, tailored, or innovative designs. This idea has been explored in previous work by Mota [15], who argues that remixing satisfies a maker's needs for new, creative, or personalized objects.
Moreover, digital content creators are often quite open about borrowing and remixing. In a study of users of howto pages, Torrey et al. [20] found that users "freely acknowledge their use of ideas they had first seen in other people's projects". By extracting valuable sub-components from existent designs and hacking them together, makers are engaging in design-despite the lack of refinement [8].

Remixing behavior has also been examined in the context of peer-produced resources including code [2,9], software configurations $[6,13,14]$, and community-authored web content such as WikiBooks [12]. However, in many ways these peer-produced resources are more straightforward than collaborating around physical products. When users share software configurations, they all configure the same interface; community-authored web content has more explicit, communal goals than the maker community; it is easier to borrow from and trace the origins of written lines of code, whereas physical features are far more difficult to specify and isolate.

Perhaps the most similar approach to ours is Cheliotis and Yew's analysis of the social structure of ccMixter [4], an online community of musicians who sample and remix each others' tracks. By analyzing the social network of remixing links between samples, Cheliotis and Yew found that merging motifs (several samples being resolved into one track) were less common than branching motifs (one track inspiring many other designs), and that site contests did not necessarily attract new users who later contributed content or remixes to the community. We use this analysis as a point of comparison with a digital media remixing network.

Finally, a few initial research efforts have started to examine peer production on Thingiverse. In particular, Papadimitriou and Paplexakis used Thingiverse metadata in the period leading up to February 2013 to look for predictive relationships between metadata features, including number of views, links, and makes [17]. They also built an interactive visualization of Thingiverse remixing ${ }^{4}$. We focus more specifically on remixing within Thingiverse, and also examine the impacts of major changes to the site in the year and a half since their analysis-including a more than 5-fold increase in the size of the site as well as the introduction of new parametric remixing tools.

\section{AN INTRODUCTION TO THINGIVERSE}

Thingiverse was founded in November 2008 by MakerBot ${ }^{5}$, a major manufacturer of consumer $3 \mathrm{D}$ printers. The site is intended to serve as a design repository and social network for hobbyist digital fabrication where community members can submit designs and share them with others. Perhaps unsurprisingly, the majority of the designs shared on the site are $3 \mathrm{D}$ models intended to be fabricated using a 3D

\footnotetext{
${ }^{4}$ http://bitquill.net/make/remix/

${ }^{5}$ http://www.makerbot.com
} 

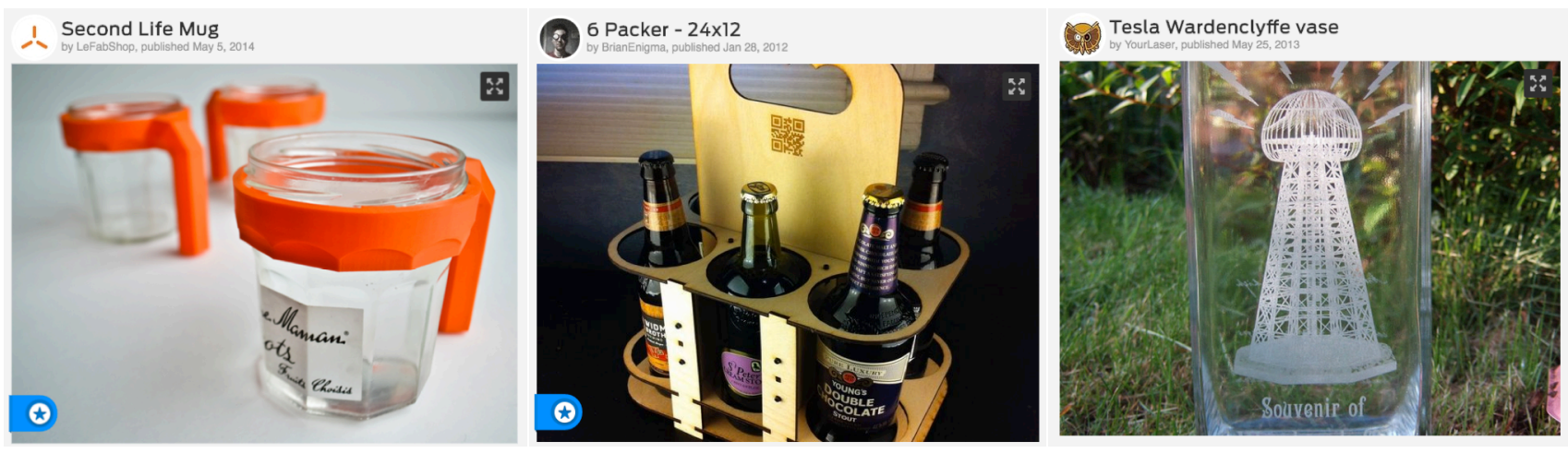

Figure 1: Things include: a) 3D designs for fabrication (3D-printed mug handle, Thing 310961, by LeFabShop); b) 2D designs for fabrication (laser-cut 6-pack holder, Thing 16643 by BrianEnigma); c) 2D designs for etching (etching on a glass vase, Thing 93972 by YourLaser, illustration by Leo Blanchette).

printer. However 2D designs for laser cutters or CNC Mills are also fairly common. The site also serves as a social network on which users can post comments, photos, and other social content.

Although many users visit the Thingiverse site, we are primarily interested in authors, i.e. those who post content under a unique username. The primary means by which authors contribute to Thingiverse is by creating Thingsdesign projects which typically include $3 \mathrm{D}$ or $2 \mathrm{D}$ design files for fabrication or etching (see Figure 1) as well images of the design and instructions for creating it. Each Thing can also include references to sources-other Things that a design is "inspired by, derived from, or a remix of". Throughout this paper, we refer to these referenced Things as sources and resulting Things as remixes.

Every Thing is assigned a unique sequential ID number when it is created and can be accessed by a unique URL that includes that ID. For example, Thing "16369" can be found at http://www.thingiverse.com/thing:16369. While they are being created, Things are private. However, once published, Things are public and are visible on the site. Authors can also delete Things and Thingiverse administrators occasionally block Things for violating the site's terms-of-service, hiding them from the site. Each public Thing page includes a range of metadata: the Thing name; the author's username; publication date; the number of times other users have liked, collected, commented, made, or remixed the design. For example, Figure 2 shows a Thing page for a "Customized Atom Deluxe" design, which can be downloaded and 3D printed.

While most Things specify a single design, some authors provide parametric models-3D designs in which some design parameters can be easily changed to generate variations of the design. During the first four years of Thingiverse, most parametric designs were shared as files from OpenSCAD 6 , a computer-assisted design (CAD) programming language. However, generating design

\footnotetext{
${ }^{6}$ http://www.openscad.org
}

variations from a parametric model required modifying the design directly in OpenSCAD.

On 15 January 2013, Thingiverse introduced Customizer ${ }^{7}$, a built-in web app that lets authors upload parametric models from OpenSCAD. Other users can then modify and generate variations directly on Thingiverse and download a customized, ready-to-print file (see Figure 3). This allows non-expert users to create new, custom designs without using code or CAD software-but this customization is limited to the parameters exposed by the original author. Customizer provides live previews of the generated design, allowing users to iterate and refine their modifications. For example, in Figure 3 a user can create a customized model atom by using a menu to select a custom typeface and using sliders to specify the size and spacing of the electron rings. Each time a user exports a generated design, it is automatically added as a new Thing with pre-populated metadata, including a name and description, instructions

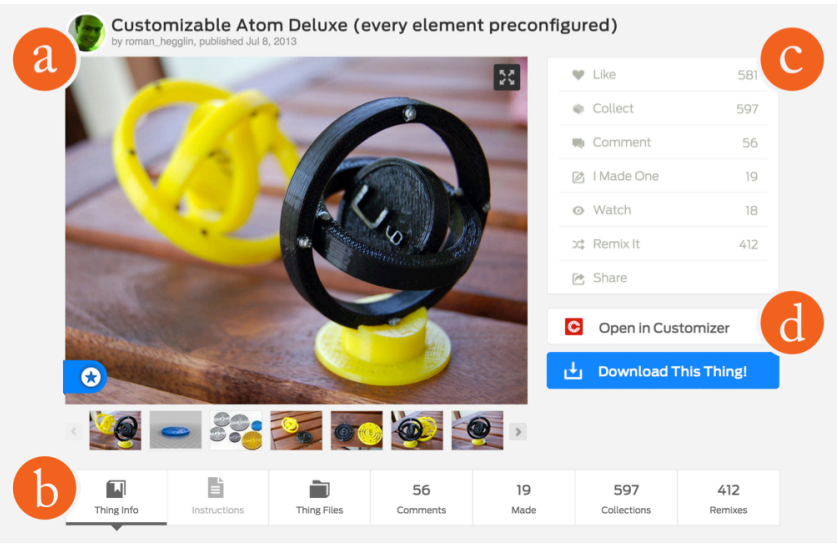

Figure 2: Customizable Atom (Thing 114247 by Roman Hegglin). Metadata includes:

a) Thing name, author's user name, publication date,

b) description, instructions, and uploaded files

c) user activity (liked, collected, commented, made, remixed)

d) link to Customizer.

${ }^{7}$ http://www.thingiverse.com/apps/customizer 


\section{Customizable Atom Deluxe (every element preconfigured)}
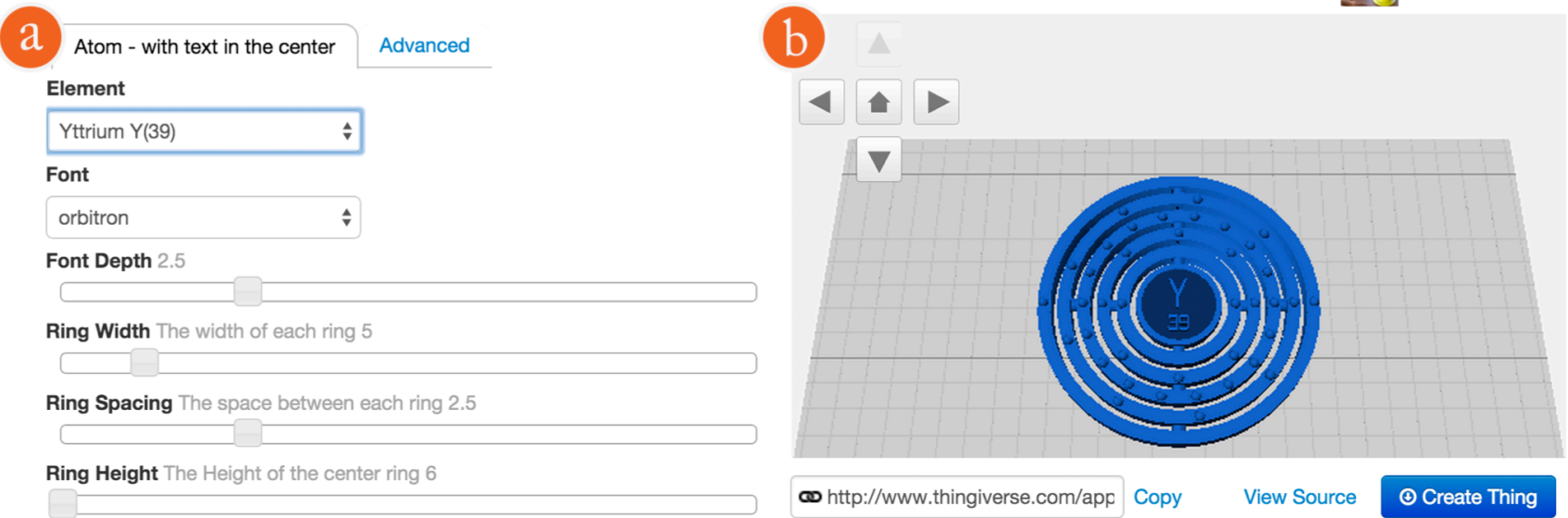

Figure 3: The Customizer App for a Customizable Atom (Thing 114247 by Roman Hegglin) allows users to a) specify font and ring characteristics, and b) preview the resulting design on a virtual model.

that contain the customization parameters, and a "customized" tag. The original parametric design is listed a source for the generated remix.

Note that all Thingiverse behavior is voluntary. Users may download and modify a design without uploading their remix to Thingiverse. Even if they do upload a remix design, they may or may not indicate the original Thing as a source. More generally, authors are free to define what constitutes a source or a remix as they create content.

\section{RESEARCH QUESTIONS}

The advent of low-cost, consumer-grade fabrication equipment has introduced an entirely new domain for remixing-specifically, the remixing of digital designs intended for physical fabrication. By examining online maker communities like Thingiverse, we can now explore this remixing behavior at a community scale. In our study we focus on the following questions:

R1. How does remixing physical designs in an online maker community differ from the remixing of other electronic media?

R2. How do parametric design tools influence authors' contribution to, and participation in online maker communities?

R3. What kinds of designs are the most influential and how can digital authoring and sharing tools foster these sorts of productive designs?

To answer these questions, we examine patterns of remixing on Thingiverse and contrast it to patterns observed in other communities, such as the audio remix site ccMixter [4]. In particular, we investigate the adoption of Customizer and how generated designs affect community behavior on Thingiverse. Finally, we analyze sources that both directly and indirectly inspire large numbers of remixes on Thingiverse and identify qualities that make them well suited for remixing.

\section{METHODOLOGY}

We conducted a quantitative analysis of Things on Thingiverse. Over the course of several days in July and August 2014, we downloaded the complete web pages for 350,354 unique Things. This corpus covers all Things published in the period from the launch of Thingiverse in 2008 until 22 July 2014, and includes data from both before and after the introduction of Customizer in January 2013. Because we collected all public Things on Thingiverse since the founding of the site, we are able to examine the evolution of how users publish and remix.

We parsed each Thing page to gather metadata about the design, including its title, author, publication date, and tags, as well as the number and types of files available for download. We also gathered information about social activity around the Thing, including the number of times that users viewed, liked, commented, downloaded, and made the Thing. Finally, we gathered a list of any source Things that the design was remixed from, and counted the number of times the design itself had been remixed. These relationships allowed us to reconstruct the complete graph of remixing on Thingiverse.

We used the Network $X^{8}$ Python library and GraphViz ${ }^{9}$ to analyze and visualize the remixing graph-links between sources and remixes that primarily exhibit a tree-like inheritance structure. We also used this data to identify particularly influential and surprising Things, which we examined via qualitative case studies.

\section{RESULTS \& DISCUSSION}

Of the 350,354 Things we collected, about half $(176,142$ Things) are public with open metadata while the other half (174,212 Things) are private or under moderation and include no metadata. This data set is several orders of

\footnotetext{
${ }^{8}$ http://networkx.github.io

${ }^{9}$ http://www.graphviz.org
} 


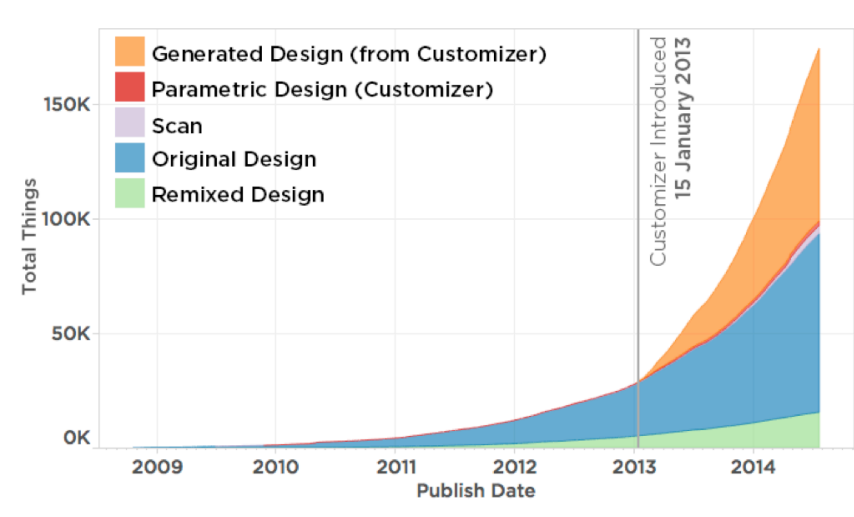

Figure 4: Total Things in Thingiverse.

magnitude larger than the content network of ccMixter which contained only 9,300 works [4]. Figure 4 shows the growth in the total number of Things in Thingiverse since its inception. This graph highlights the impact of Customizer, whose introduction in January 2013 corresponds with a huge jump in activity. The total number of Things on the site nearly tripled in the following year, and generated designs from Customizer now represent an increasingly large fraction of total designs.

\section{Authorship}

Since the introduction of Customizer, the number of authors contributing content to Thingiverse has grown by almost $600 \%$, from 7,230 authors on 15 January 2013 to 42,957 by 22 July 2014. Most authors (39,722 or 92\%) publish fewer than 10 Things $($ median $=2$, average $=4$ ), and 19,357 authors (45\%) published only one Thing. On average, authors with more than one Thing were active for 144 days (median $=39$ days). We divided authors into three groups, based on when they were active on Thingiverse:

- Authors who were only active before the introduction of Customizer (4,678 authors or $11 \%)$.

- Authors who are active both before and after the introduction of Customizer (2,531 authors or 6\%).

- Authors who are only active after the introduction of Customizer (35,749 authors or $83 \%)$.

While many authors who contribute Things were never involved in remixing $(13,500$ or $31 \%)$, we are most interested in the 29,458 authors (69\%) who contributed either sources or remixes. This fraction is lower than the number of authors (79\%) who were involved in remixing on ccMixter in Cheliotis and Yew's analysis [4]. One possible explanation is that Thingiverse places more emphasis on sharing content for fabrication, while ccMixter is expressly focused on remixing.

Of the authors who were involved in remixing, the majority $(24,382$ or $83 \%)$ only remixed others' work and their work never served as the source for a remix. A smaller number $(3,285$ or $11 \%)$ both created remixes and had their work remixed by others, while an even smaller minority $(1,791$ or
$6 \%$ ) had their work remixed by others but did not create remixes themselves.

\section{Remixing Graph}

We created a directed graph of remixing relationships between Things, where vertices represent the 98,766 Things involved in remixing. Edges in the graph represent remix relationships and point from source Things to the Things that remix them. This created a number of tree-like subgraphs in which the provenance of each Thing can be traced back over multiple generations of prior remixes. We measured the generation of each remixed Thing by counting the path length between it and its most distant ancestor.

Many of the motifs (see Figure 5) identified by Cheliotis and Yew [4] in music remixing also appear in our graph and correspond with common design activities. For example, branching out from a source indicates divergent ideation of new designs, while merging integrates multiple sources of inspiration into one remix design.

The entire remixing graph consists of 6,536 independent subgraphs. The majority of these subgraphs $(5,187$ or $79 \%)$ only include one generation of remixing. Most subgraphs $(3,760$ or $58 \%)$ are pairs-one isolated source with a single remix. Many other subgraphs $(1,427$ or $22 \%)$ are simple examples of branching and merging patterns. For example, 1,297 subgraphs are source stars (average \# remixes $=12$; median $=3, \max =832$ ), in which one generation of remixes emanates from a single source Thing. The majority of these source stars (724 subgraphs or 55\%) were created from Customizer. Meanwhile, 130 subgraphs are remix stars (average \# sources $=2$; median $=2 ; \max =13$ ) in which one generation of sources are combined in a single remix. In total, 16,860 Things (17\% of the remixing graph) were sources or remixes in star structures.

However, as in electronic media remixing, remixing in Thingiverse also exhibits a variety of more complex patterns. Over $20 \%$ of subgraphs (1349 of 6,536), comprising 74,386 nodes, contain complex combinations remixing patterns. In these remaining subgraphs, we saw a number of anomalous motifs such as mutual citation dyads (114 occurrences), feed-forward loops (1,713 occurrences), and even one self-loop (see Figure 5). These often appear to be evidence of a lack of support for more nuanced tracking of versions and relationships among Things. For example, Thing 13710 referenced itself as its own source, creating a self-loop. In fact, the author edited this Thing to post an updated version of his or her original design, noting "version 2 of the original logo" in the description.



PAIR

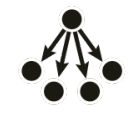

SOURCE

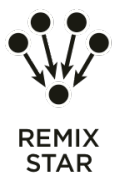

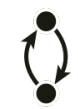

MUTUAL

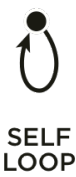

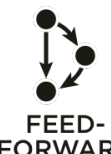

FORWAR

Figure 5: Common network motifs in Remixing Graph. 


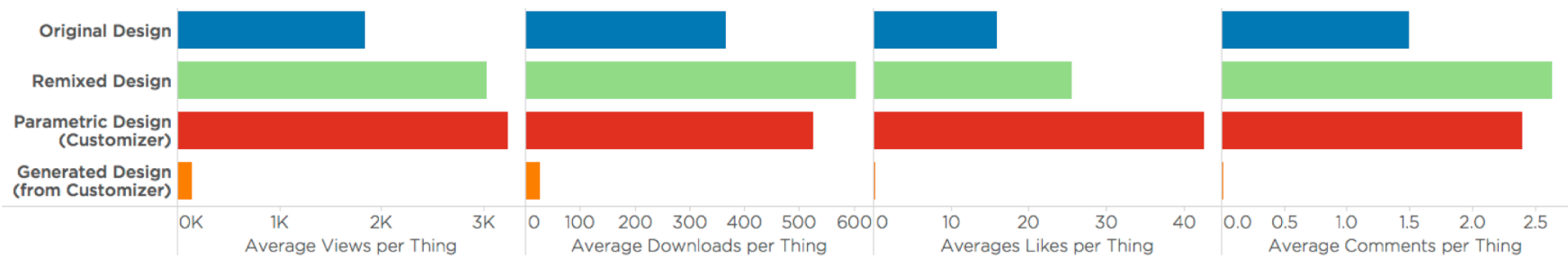

Figure 6: Average User Activity level (Views, Downloads, Likes, and Comments) by Thing Category. Very few generated designs from Customizer elicit subsequent user activity, in contrast to all other types of Things.

Ultimately, Things can be remixed in highly diverse ways and there is no single archetypal pattern. The one clear exception, however, is Customizer, where remixing manifests itself very differently.

\section{Parametric Design Tools \& Customization}

The number of Things in Thingiverse has risen steeply since the introduction of Customizer (see Figure 4). As of July 2014, the site contains 1,692 parametric designs that are configured for use with Customizer. These make up only $2 \%$ of the remixing graph and fewer than $1 \%$ of all Things on the site. However, authors have used Customizer to generate 74,949 new Things from these parametric designs. These generated Things now make up $74 \%$ of the remixing graph and $42 \%$ of all public Things on the site. Thus the majority of remixed Things are derived directly from a very small number of parametric designs. In fact, the top 120 most-remixed Things are all parametric designs from Customizer, each of which has been used to produce hundreds or thousands of generated designs. The most popular Customizer designs (including 9 of the top 10 most-remixed Things) allow users to add or change aesthetic features-primarily by adding text or images to an existing object. These aesthetic parametric models are classic examples of mass-customization and personalization but they provide few opportunities to tailor designs to new contexts or improve their functionality.

Although designs generated from Customizer make up the majority of the remixing graph, they are mostly dead-ends and do not inspire subsequent user engagement. Of the 74,958 generated Things, only 59 are sources with remixes of their own! Generated designs also prompt almost no additional user activity and-as Figure 6 shows-they are rarely viewed, downloaded, liked, or discussed.

Generated designs themselves do not seem to contribute to the broader Thingiverse community. However, one hypothesis might be that creating generated designs can still inspire authors to participate more broadly and eventually create other remixes or original designs. To test this, we calculated the proportion of authors' total things that were generated using Customizer, focusing specifically on authors who started using Thingiverse after Customizer's introduction. We then sorted authors who had created at least two Things into one of three categories: those who only created generated designs with Customizer, those who never used Customizer, and those who both used Customizer and created remixed or original Things.

Figure 7 shows that $49 \%(9,101)$ of these authors only generated designs using Customizer, while a similar number $(6,397$ or $39 \%)$ never used Customizer. Surprisingly, only $17 \%(3,199)$ of these authors generated designs in Customizer and created outside of Customizer.

While this split is influenced by the fact that the majority of authors on Thingiverse $(35,527$ or $83 \%)$ have created less than 5 things, a divide persists even among much more active authors. For example, among the 148 authors with 25-29 Things, 42\% (63 authors) still exclusively generated designs using Customizer, and 28\% (42 authors) had never used it. This divide suggests that Customizer may have attracted a large, new group of authors to Thingiverse, but that few of these authors transition from using Customizer to creating their own designs or remixing without the support of parametric designs. Meanwhile, a separate large group of authors avoid Customizer completely.

\section{Successful Sources of Remixing}

We argue that generating a design by simply manipulating prescribed parameters does not fit with the more experimental and creative vision of remixing. To seek out remixing behavior that more closely resembles this vision, we delved deeper into the $23 \%$ of the remixing graph $(23,125$ Things, $13 \%$ of all public Things) that do not involve Customizer. In particular, we wanted to identify what common types of source Things successfully inspire new and different designs or have lasting impact.

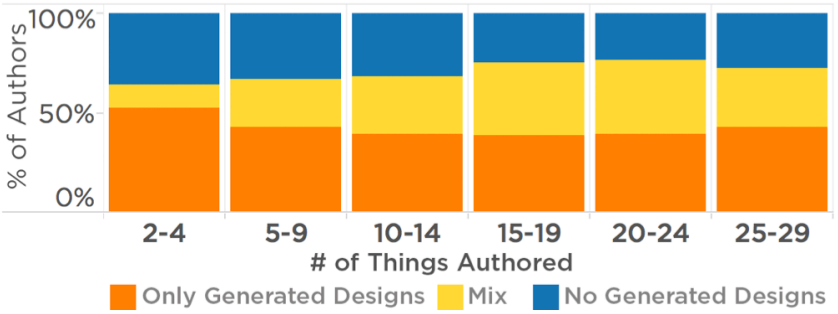

Figure 7: Percent of authors who have only generated designs from Customizer (orange-bottom), who have no Customizer-generated designs (blue-top), and who have a mix (yellow-middle), by the author's total number of Things.

Only includes authors who started using Thingiverse after the introduction of Customizer. 
To identify successful sources, we excluded all Things generated with Customizer from our dataset and calculated three measures of influence: number of remixes, number of descendants, and generations of influence. We then manually examined the 40 sources with the most remixes, the 40 sources with the most descendants, and the 40 longest remix chains. We thematically clustered these Things and chains to identify characteristics that may lead Things to become influential sources. We identified four genres of successful Things:

Assemblies and their Components: Many authors post complex assemblies of parts. Sometimes assemblies are posted as a single Thing, while other times individual parts are posted separately. Both assemblies and their components are influential in the remixing graph, with authors often borrowing pieces from a source or combining individual sources into assemblies. Assemblies often correspond to directed or well-defined projects. For example, the DIY 3D Printer community dominates most measures of successful remixing with 27 of the mostremixed sources and 32 of the sources with the most descendants. It is also the subject of 14 of the longest remix chains. Another example of a successful assembly is flying quadcopters, with 2 of the most-remixed sources. These directed projects attract a wide range of contributors-full assemblies of the Rostock (Thing 17175) and MendelMax (Thing 20355) DIY 3D printers each had over 60 remixes from 41 unique authors. These examples clearly illustrate the type of remixing advocated by Anderson [1], in which a group of authors collaborate and iterate on each others' designs.

Primitive Mechanisms and Forms. Some influential Things are primitive mechanisms ( 2 of the most-remixed sources and 6 of the sources with the most descendants) or primitive forms ( 7 of the most-remixed sources and 9 of the sources with the most descendants). Successful primitive mechanisms such as screws (Thing 8796; Thing 8793) or pin connectors (Thing 10541) are applicable in many domains. These primitives are usually remixed to add a specific functionality or aesthetic properties to an object.

Meanwhile, successful primitive forms range from basic shapes like a 3D heart (Thing 6190) to small sculptural figurines that can easily be fused or attached to other models. Many of these forms, including a "Gangsta" figurine (Thing 5367), "Beefy Arms" (Thing 217849,), a Cute Octopus (Thing 7900), and a scanned bust of comedian Stephen Colbert (Thing 9104) have become memes on Thingiverse and are often remixed as in-jokes within the community.

Sets of Things: Some Things appear influential because they are representative of a set-a group of independent Things that are thematically related. For example Thingiverse contains at least 33 different pieces that can be printed and combined to build marble runs. All of these pieces are posted as separate Things, but are listed as

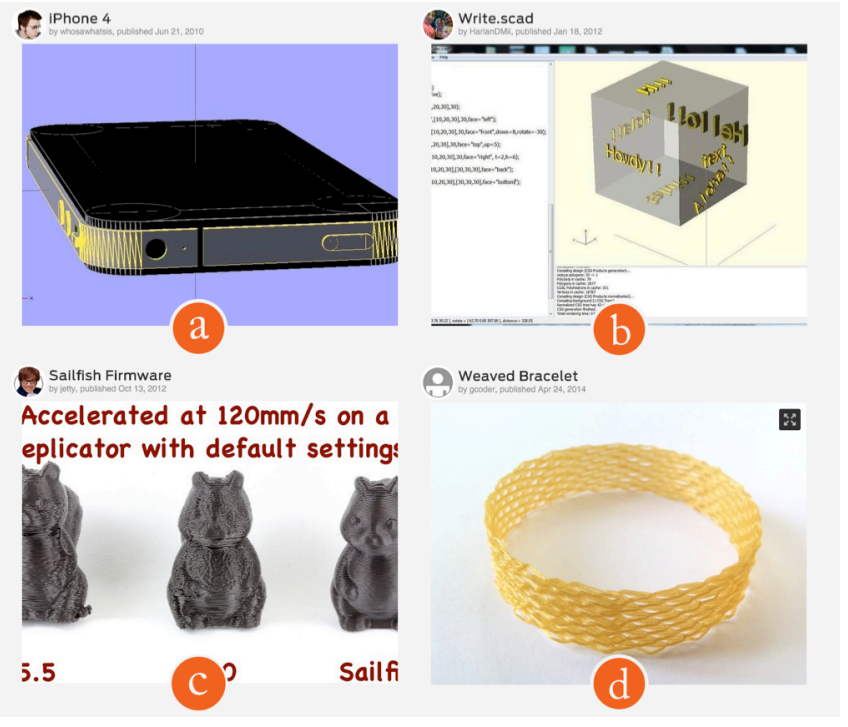

Figure 8: Non-physical Things include: (a) a reference model for making iPhone accessories (Thing 3392 by whosawhatsis), (b) software library for a CAD tool (Thing 16193 by HarlanDMii), (c) firmware that improves 3D print quality (Thing 32084 by jetty), and (d) novel fabrication technique demonstrated with a simple aesthetic example (Thing 309548 by gcoder).

remixes of the same original piece (Thing 62314). This remixing relationship is the main indicator that identifies them as a set. Unlike assemblies, Things in a set tend to be remixed by the author of the original source. In fact, three out of four sets in our sample were exclusively remixed by the source author.

Accessories: Another influential class of sources is aftermarket accessories for consumer products. Remixing here may be useful because accessories require careful physical fit with an existing, often mass-produced object. For example, two of the most-remixed sources in our sample are both enclosures for a Raspberry Pi computer (Thing 16104; Thing 24461). Starting from designs allows remix authors to keep the geometry that interfaced with the computer itself while tailoring the case to their own needs. We also observed chains of remixes that modify Things designed to work with one type of device to fit another. For example, in one remix chain, a mount protector designed for an SLR camera (Thing 11780) is remixed over several generations to hold a GPS, fit a different camera, and ultimately interface with a GoPro.

\section{Non-Things: Tools \& Techniques}

Thingiverse's guidelines specify that "designs must represent a real, physical object that can be made." However, while exploring influential sources, we noticed that many source Things do not represent physical objects at all. To more deeply examine the role of non-physical Things in Thingiverse, we used snowball sampling to gather a set of 55 examples. We clustered these Things according to their roles and discovered that most represent 
various Tools and Techniques that support design and fabrication processes:

Design Software. We found 16 examples of standalone software and plug-ins for CAD tools. These software tools address a variety of computational needs, including giving users increased control over surfaces in digital models (Thing 8786) and converting 2D shapes into 3D forms (Thing 24639, Thing 25036). By most measures, the most influential Thing in Thingiverse is Write.scad() (Thing 16193, Figure 8b)-a library for OpenSCAD that allows designers to add extruded text to models. This library has been directly remixed more than any other Thing (105 remixes). Moreover, because many of its total remixes are parametric Customizer designs it has ultimately influenced a very large number of total descendants (14,276).

Reference Models. Some Things contain 3D models but are not intended for fabrication. We found 13 examples of reference models whose geometry provides accurate measurements for a design. Our sample of reference models primarily consists of popular consumer electronics (e.g., mobile phones, cameras) and kit electronics (e.g., Raspberry Pi, Arduino, Adafruit Flora) and can be used to build accessories for them. For example, Figure 8a (Thing 12879) shows a precise model of an iPhone based on published specifications from Apple. Comments on these models indicate that authors use them to ensure correct fiteither by using the model in CAD software or, in rare cases, by printing the model to physically test it.

Design Workflows. We also found 7 examples of workflows that explain processes for connecting existing design software in order to produce new designs. For example, "Huge Yosemite Model" (Thing 351271) offers workflow instructions on how to create 3D models from LIDAR data. These workflows offer step-by-step instructions more commonly found in how-to pages [20].

Fabrication Firmware. Makers can augment their fabrication machines by adapting or improving the firmware that drives them. We found 9 different firmware packages, the most popular of which was Sailfish (Thing 32084, Figure 8c) a $3 \mathrm{D}$ printer firmware that offers finer resolution and better quality output.

Fabrication Techniques. Makers can use low-level programming languages to control specific fabrication parameters. For example, GCode scripts can control tool paths, extrusion temperatures, and feed rates for 3D printers. These scripts allow makers to invent new ways of manipulating material to create forms and structures. We found 10 examples, including the Weaved Bracelet (Thing 309548, see Figure 8d)-a GCode script that creates a bracelet "woven" by a 3D printer. In another example, Laser Origami (Thing 180990) replicates a fabrication technique originally introduced by Mueller et al. [16]. While fabrication techniques sometimes appear as independent non-physical Things, they are often presented

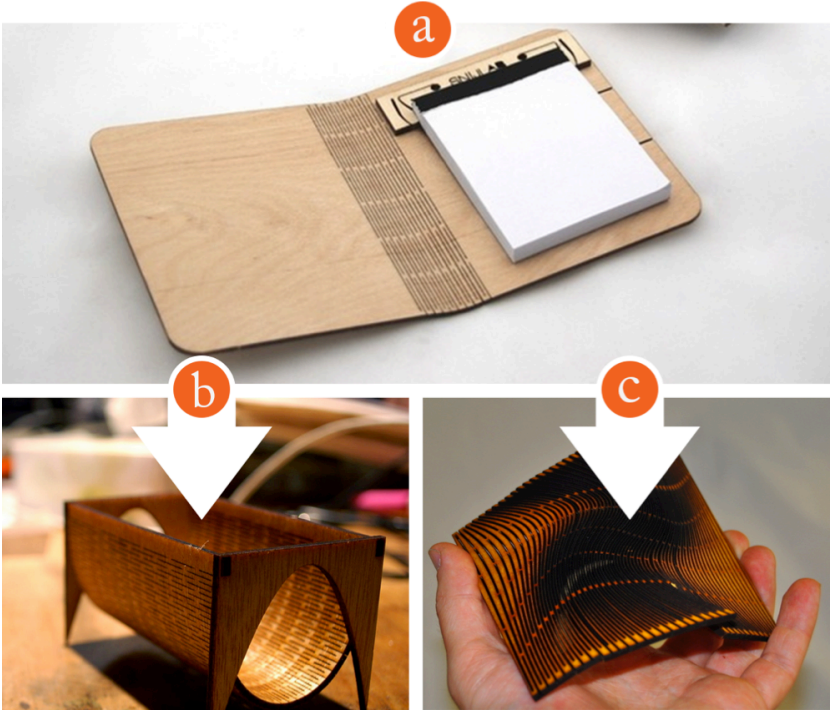

Figure 9: The a) Folding Wood Booklet (Thing 12707 by snijlab) shows a novel hinge technique from laser-cut plywood. It was remixed into a b) Business card holder (Thing 15845 by Guy Dickinson) as well as c) Test pieces for exploring forms made possible with this technique (Thing 12859 by Beehive).

in the context of an example application. Subsequent remixes may abstract the technique to help others apply it for new designs.

\section{Remixing Non-Physical Things}

Authors may remix tools and techniques either to improve or apply them. Improved versions of tools may be more efficient than the original source, add new functionality, or update the original to work with more recent physical objects, fabrication machines, or software. For example, the Folding Wood Booklet (Thing 12707, Figure 9a) presents a laser-cut integrated living hinge, and has been remixed in multiple ways. Some remixes (e.g., Thing 12859, Figure 9c) improve and extend the technique itself by creating test shapes that explore the range of flexible forms. Alternatively, the same tools or techniques can be applied to create specific designs (e.g., Thing 15845, Figure 9b).

Others may appropriate the tool or technique in unintended or unexpected ways. For example, we found a series of Things (e.g., Thing 13620) that use GCode to control the motors in a $3 \mathrm{D}$ printer and create musical sound patterns. By applying the tool in a radically different way, authors who appropriate introduce new possible functionalities to the tool, without modifying the tool itself.

Other examples represent the creative reframing of the concept of a Thing as a performance of making that can be recreated, shared and appropriated by others in the maker community. For example, Thing 40770 provides not only the design of a new cup, but also the concept of creating a new cup design every week. Each week the author created a new cup but marked it as a remix of the original cup. This link connected subsequent designs to the routine that 
produced them. In cases like this, the source does not provide any reference geometry, but inspiration on how to perform making on a regular basis.

\section{IMPLICATIONS FOR DESIGN}

Based on our examination of Thingiverse, it is clear that previous notions of remixing in maker communities are incomplete. While parametric tools like Customizer allow more users to generate personalized objects, these designs end up isolated from the rest of Thingiverse. Moreover the most influential sources for remixing include both physical things like assemblies, primitive forms, and mechanisms and non-physical things-tools and techniques that aid design and fabrication. Building on these findings we identify opportunities for online maker communities to foster better remixing practices.

\section{Tracking Versions and Assemblies}

Version tracking and support for specifying assembly relationships between parts are both already found in PDM systems such as Autodesk Vault ${ }^{10}$. However, on Thingiverse remixing is the only systematic way of indicating a relationship between things. Adding version tracking and assembly support features to an online maker community could allow makers to collaborate on more complex assemblies and avoid the confusion caused by multiple versions of the same design.

\section{Making the Remixing Graph More Accessible}

In our experience as researchers, our visual representations of the remixing graph immediately clarified how specific Things related to and influenced each other. Currently, navigating the graph of remixed and related designs on sites like Thingiverse tends to be linear and tedious, with only one generation of designs visible at a time. Makers could benefit from browsing and search tools that allow them to more fluidly traverse the remixing graph to find influential or readily remixable sources. By exploring families of related designs, authors could better understand the relationships between designs and select the best sources for their own remixes.

\section{Providing Reference Models for Consumer Products}

Accessories for consumer products are popular on Thingiverse because they allow makers to personalize or extend the utility of their existing devices. Despite this enthusiasm, makers manually create almost all reference models for devices or attachment points. Companies and standards bodies could support user engagement by systematically posting reference models of products and standard parts to online maker communities-actively encouraging users to build accessories that enhance consumer products. Remixes that utilize these models would presumably be more reliable because they are based on accurate, verified geometry. Moreover, authors could easily locate accessories for an object by looking for designs that utilize its reference model.

\section{Highlighting Tools \& Techniques}

While Thingiverse requests that new Things "real, physical object that can be made", non-physical tools and techniques offer unique value to users as they are remixed and applied to new designs. Online maker communities need to highlight non-physical tools and techniques so that authors can more quickly and creatively develop, adapt, or customize their designs.

\section{Learning Design through Parametric Models}

A large number of authors on Thingiverse only produce generated designs using Customizer and never contribute other content. One possible reason for this is that many authors lack the technical expertise necessary to modify designs using CAD tools. One way to bridge this gap might be to allow authors to open parametric designs like those used in Customizer in beginner-friendly CAD tools, and generate designs from there. This could provide a less intimidating entry point for CAD where users could continue to interact with and refine designs according to its predefined parameters. These tools could help scaffold users by showing them alternative ways of making the same changes to models using the CAD interface. Over time, as users become more familiar with CAD, they could not only adjust parameters but also begin to add new features, creating richer remixes and building their expertise. Ultimately this could scaffold users' development as designers, using an approach similar to Carle's Virtual Design Apprenticeship model [3]: adopt, adapt, combine, and create.

\section{Aggregating Users' Experiences}

When authors generate designs using Customizer, they typically have little or no information about which parameterized settings are good or effective combinations. This is true, even if hundreds or thousands of others have attempted to generate the same design. Online maker communities with parametric design tools could improve this experience by tracking how the community uses these tools, and displaying this data back to authors. For example, tools like Customizer could summarize previous users' choices using small charts integrated into the dropdowns and sliders used to specify parameters [22]. These social cues could help authors identify and replicate common configurations or produce new unique designs.

A similar approach could be used to share successful or unsuccessful fabrication experiences. When producing a design, authors often have little information about whether or not it will work with their fabrication equipment, materials, or machine settings. Online maker communities could help fill this gap by rewarding authors who document their experience of fabricating a design and contribute information about the configuration they used. This information could highlight tested fabrication setups and discourage authors from repeating others' failed attempts.

\footnotetext{
${ }^{10}$ http://www.autodesk.com/vault
} 


\section{CONCLUSION AND FUTURE WORK}

This paper provides the first community-scale analysis of remixing behavior in online maker communities. Our analysis shows that authors of 3D designs on Thingiverse do not remix as frequently or draw from as many sources as those in online audio communities like ccMixter. We also characterized the impact of parametric design tools like Customizer, which provide lightweight ways of producing generated designs. We find that Customizer falls short of traditional definitions of remixing, in that generated designs do not elicit subsequent user activity and relatively few authors create both generated designs and original ones.

Outside of Customizer, we do find examples of productive, influential sources of remixing in the form of complex assemblies, accessories, primitive mechanisms, and primitive forms. However, some non-physical things are also influential sources of remixing. In fact, we found numerous examples of non-physical Things that support design and fabrication process by providing reusable tools and techniques.

Ultimately, online maker communities document the performance of making, and allow authors to recreate or find inspiration in others' making experiences. Thingiverse is by no means the only online maker community that supports remixing. Other sites like Ravelry and Autodesk's 123D Circuits ${ }^{11}$ formalize remixing using different (and sometimes more expressive) mechanisms and may provide a useful counterpoint. Thus, comparing remixing behavior across these sites could help us even better understand how the design of maker communities impacts creative output.

\section{ACKNOWLEDGMENTS}

We would like to thank the Thingiverse authors who gave permission to use images. This research was supported by the European Research Council under grant ERC-2012AdG \#321135 CREATIV.

\section{REFERENCES}

1. Anderson, C. Makers: The New Industrial Revolution. Crown Business, New York, 2012.

2. Biazzini, M. and Baudry, B. "May the Fork Be with You": Novel Metrics to Analyze Collaboration on GitHub. Proc. of International Workshop on Emerging Trends in Software Metrics, ACM (2014), 37-43.

3. Carle, A. Promoting Learning of Instructional Design via Overlay Design Tools. 2012.

http://www.eecs.berkeley.edu/Pubs/TechRpts/2012/EEC S-2012-246.pdf.

4. Cheliotis, G. and Yew, J. An Analysis of the Social Structure of Remix Culture. Proc. of Conference on Communities \& Technologies, ACM (2009), 165-174.

5. Dougherty, D. The Maker Movement. Innovations: Technology, Governance, Globalization 7, 3 (2012), 1114.

\footnotetext{
${ }^{11}$ http://123d.circuits.io
}

6. Gantt, M. and Nardi, B.A. Gardeners and Gurus: Patterns of Cooperation Among CAD Users. Proc. of CHI 1992, ACM (1992), 107-117.

7. Goodman, E. and Rosner, D. From Garments to Gardens: Negotiating Material Relationships Online and 'by Hand.' Proc. of CHI 2011, ACM (2011), 22572266.

8. Hartmann, B., Doorley, S., and Klemmer, S.R. Hacking, Mashing, Gluing: Understanding Opportunistic Design. IEEE Pervasive Computing 7, 3 (2008), 46-54.

9. Hill, B.M. and Monroy-Hernández, A. The Cost of Collaboration for Code and Art: Evidence from a Remixing Community. Proc. of CSCW 2013, ACM (2013), 1035-1046.

10.Kuznetsov, S. and Paulos, E. Rise of the Expert Amateur: DIY Projects, Communities, and Cultures. Proc. of NordiCHI, ACM (2010), 295-304.

11.Lessig, L. Remix: Making Art and Commerce Thrive in the Hybrid Economy. Penguin Books, New York, 2009.

12.Liccardi, I., Chapuis, O., Yeung, C.-M.A., and Mackay, W. Redundancy and Collaboration in Wikibooks. In P. Campos, N. Graham, J. Jorge, N. Nunes, P. Palanque and M. Winckler, eds., Human-Computer InteractionINTERACT 2011. Springer Berlin Heidelberg, 2011, 215-232.

13. Mackay, W.E. Patterns of Sharing Customizable Software. Proc. of CSCW 1990, ACM (1990), 209-221.

14.Mackay, W.E. Triggers and Barriers to Customizing Software. Proc. of CHI 1991, ACM (1991), 153-160.

15. Mota, C. The rise of personal fabrication. Proc. Creativity \& Cognition 2011, ACM (2011), 279-288.

16. Mueller, S., Kruck, B., and Baudisch, P. LaserOrigami: Laser-cutting 3D Objects. Proc. of CHI 2013, ACM (2013), 2585-2592.

17.Papadimitriou, S. and Papalexakis, E.E. Towards Laws of the 3D-printable Design Web. Proc. of ACM Web Science, ACM (2014), 255-256.

18. Rosner, D. and Bean, J. Learning from IKEA Hacking: I'm Not One to Decoupage a Tabletop and Call It a Day. Proc. of CHI 2009, ACM (2009), 419-422.

19. Tanenbaum, J.G., Williams, A.M., Desjardins, A., and Tanenbaum, K. Democratizing Technology: Pleasure, Utility and Expressiveness in DIY and Maker Practice. Proc. of CHI 2013, ACM (2013), 2603-2612.

20.Torrey, C., McDonald, D.W., Schilit, B.N., and Bly, S. How-To pages: Informal systems of expertise sharing. In L.J. Bannon, I. Wagner, C. Gutwin, R.H.R. Harper and K. Schmidt, eds., ECSCW 2007. Springer London, 2007, 391-410.

21. Wakkary, R. and Maestri, L. The Resourcefulness of Everyday Design. Proc. of Creativity \& Cognition 2007, ACM (2007), 163-172.

22.Willett, W., Heer, J., and Agrawala, M. Scented Widgets: Improving Navigation Cues with Embedded Visualizations. IEEE Transactions on Visualization and Computer Graphics 13, 6 (2007), 1129-1136. 